\title{
Group decision-making approach for flood vulnerability identification using the fuzzy VIKOR method
}

\author{
G. Lee ${ }^{1}$, K. S. Jun ${ }^{2}$, and E.-S. Chung ${ }^{3}$ \\ ${ }^{1}$ Department of Water Resources, Graduate School of Water Resources, Sungkyunkwan University, Suwon, South Korea \\ ${ }^{2}$ Department of Water Resources, Graduate School of Water Resources, Sungkyunkwan University, Suwon, South Korea \\ ${ }^{3}$ Department of Civil Engineering, Seoul National University of Science and Technology, Seoul, South Korea
}

Correspondence to: E.-S. Chung (eschung@ seoultech.ac.kr)

Received: 1 July 2014 - Published in Nat. Hazards Earth Syst. Sci. Discuss.: 30 September 2014

Revised: 7 February 2015 - Accepted: 11 March 2015 - Published: 20 April 2015

\begin{abstract}
This study proposes an improved group decision making (GDM) framework that combines the VIKOR method with data fuzzification to quantify the spatial flood vulnerability including multiple criteria. In general, GDM method is an effective tool for formulating a compromise solution that involves various decision makers since various stakeholders may have different perspectives on their flood risk/vulnerability management responses. The GDM approach is designed to achieve consensus building that reflects the viewpoints of each participant. The fuzzy VIKOR method was developed to solve multi-criteria decision making (MCDM) problems with conflicting and noncommensurable criteria. This comprising method can be used to obtain a nearly ideal solution according to all established criteria. This approach effectively can propose some compromising decisions by combining the GDM method and fuzzy VIKOR method. The spatial flood vulnerability of the southern Han River using the GDM approach combined with the fuzzy VIKOR method was compared with the spatial flood vulnerability using general MCDM methods, such as the fuzzy TOPSIS and classical GDM methods (i.e., Borda, Condorcet, and Copeland). As a result, the proposed fuzzy GDM approach can reduce the uncertainty in the data confidence and weight derivation techniques. Thus, the combination of the GDM approach with the fuzzy VIKOR method can provide robust prioritization because it actively reflects the opinions of various groups and considers uncertainty in the input data.
\end{abstract}

\section{Introduction}

In South Korea, flood mitigation policy is not controlled by only one organization. The Ministry of Land, Infrastructure, Transport and Tourism (MLIT) has developed the comprehensive water resources plan of large dams and rivers to mitigate the flood damage and protect people's properties and damages. The Ministry of Security and Public Administration (MOSPA) has taken responsibility of small rivers and their flood damages. Local governments have to recover the damages when flood damage arises. When flood occurs or any flood mitigation projects are determined, conflicts among by MLIT, MOSPA and local governments are frequently taken place. Therefore, effective decision making system must be necessary to incorporate different opinions from various stakeholders.

Many studies have indicated that stakeholder involvement is crucial to vulnerability assessments. According to the Danube Floodrisk project (2012), individual and institutional knowledge and the expertise of stakeholders are decisive factors in flood damage adaptation and risk assessment. However, the majority of stakeholders, including policymakers, communities, NGOs, engineers, and civil servants, have differing opinions, which are shaped by their personal experiences and areas of interest, such as social, economic, political, engineering, and environmental issues. Thus, flood vulnerability can be effectively identified if multi-criteria decision-making (MCDM) techniques are employed to aggregate various opinions and factors. MCDM can incorporate qualitative as well as quantitative data, which may be 
conflicting and uncertain, based on the aggregated opinions of all stakeholders.

However, flood vulnerability identification is complicated by the increase in the number of decision criteria and involved groups; that is, flood vulnerability assessment is inevitably associated with a noticeable degree of uncertainty (Jun et al., 2013; Lee et al., 2013) that can be categorized as follows: (1) uncertainty due to the vagueness of data and (2) uncertainty caused by the subjective viewpoint of the decision maker. The first type of uncertainty is related to the precise data measurement and composition for the spatial and temporal values of social, economic, and hydrologic consequences, such as population, property value, and flood inundation area. The second type of uncertainty is caused by the differences among the personal characteristics of each decision maker. The uncertainty caused by the subjective viewpoint of the decision maker means that the decision maker's opinion has been changed by their knowledge. If the decision maker learns more about the conditions such as the possibility of occurrence of a potentially damaging natural event and the change of socioeconomic vulnerable factor in a given area, their view point would be changed. Therefore, the main challenges in flood vulnerability identification are both reducing the uncertainty of the crisp data of proxy variables and the uncertainty of subjectively-driven weights.

For the first uncertainty, some studies have combined MCDM methods with fuzzy set theory to address various uncertainties because fuzzy MCDM methods reduce the uncertainty of parametric approaches, which is inherent to weight determination and the derivation of crisp input data. These methods have been employed in various fields, such as groundwater vulnerability (Zhou et al., 1999), tourism (Tsaur, 2002), plant location selection (Chu, 2002), robot selection (Chu and Lin, 2003), water resources management (Kim et al., 2013; Kim and Chung, 2013a, 2014; Chung and Kim, 2014), group decision making (Shih et al., 2007), reservoir operation ( $\mathrm{Fu}, 2008$; Afshar et al., 2011), knowledge management (Chang and Wang, 2009), the airline industry (Torlak et al., 2011), and environmental watershed planning (Chen et al., 2011). This study also utilized triangular fuzzy numbers (TFNs) to determine the criteria and their corresponding weights. Fuzzy numbers effectively reduce the first type of uncertainty.

The second type of uncertainty is caused by disagreements on the issue. In the case of decision making for flood management, stakeholders have different perceptions about flood hazards, which reflect their different socioeconomic and sociopsychological backgrounds. Mutual frictions between the stakeholders, which are based on different goals and objectives, should be recognized and considered to determine the most preferred alternative using the preferences of individual decision makers. The group decision-making (GDM) method coupled with MCDM has also been proven to be an effective tool that enables a converging and compromising opinion for decision makers (Speller, 2005). The GDM method is an ap- proach in which an entire group of people can participate in consensus building. The method enables stakeholders to make better decisions using various information and knowledge of the decision makers. It has the advantage of sharing the responsibility for the consequences. However, many difficulties arise in the transition from a single-decision-maker process to a multiple-decision-maker-driven process. The latter becomes more complicated due to an increase in the number of individuals/groups (stakeholders) involved in the decision-making process (Akter and Simonovic, 2005). Various methods that efficiently gather the opinions of each decision maker by subjective evaluation have been investigated to address this issue. Classic GDM methods, such as the Borda (William, 1978), Condorcet (McLean, 1990), and Copeland (1951) methods, employ the voting rule. Each member of the group scores alternatives in order of relative preference. However, these methods are not concerned with the difference in the evaluated value because they only rank the alternatives by the opinions of the decision maker. A recent refinement of the GDM method focused on the integration of a new MCDM technique. Kim and Chung (2013b) combined the fuzzy-based Technique for Order of Preference by Similarity to Ideal Solution (TOPSIS) method and voting methods to evaluate the climate change vulnerability of water resource systems.

Therefore, this study developed an approach to assess flood vulnerability using the fuzzy-based VIKOR method for GDM. The VIKOR (VlseKriterijumska Optimizacija I Kompromisno Resenje in Serbian) method is another MCDM method that employs aggregating functions; it focuses on compromising solutions for a prioritization problem with conflicting criteria, which can help decision makers obtain final solutions (Opricovic and Tzeng,2007). This approach was subsequently employed to quantify the flood vulnerability of the southern Han River basin in South Korea where Lee et al. (2013) used fuzzy TOPSIS and the average weights of decision makers to identify the flood vulnerability for the reduction of the crisp data uncertainty. However, this study showed a new framework to improve the limitation of the previous study which has not considered the weight differences among all stakeholders by introducing the fuzzy VIKOR method for GDM.

\section{Methodology}

\subsection{Study procedure}

This study consisted of four steps, as shown in Fig. 1. The first step is to identify the criteria of flood vulnerability and their weight sets using the Delphi survey because floods have a significant influence on society. In this study, flood vulnerability criteria were constructed using a hierarchy tree that consists of social, economic, and hydrologic components, which indicate the causal relationship between society 


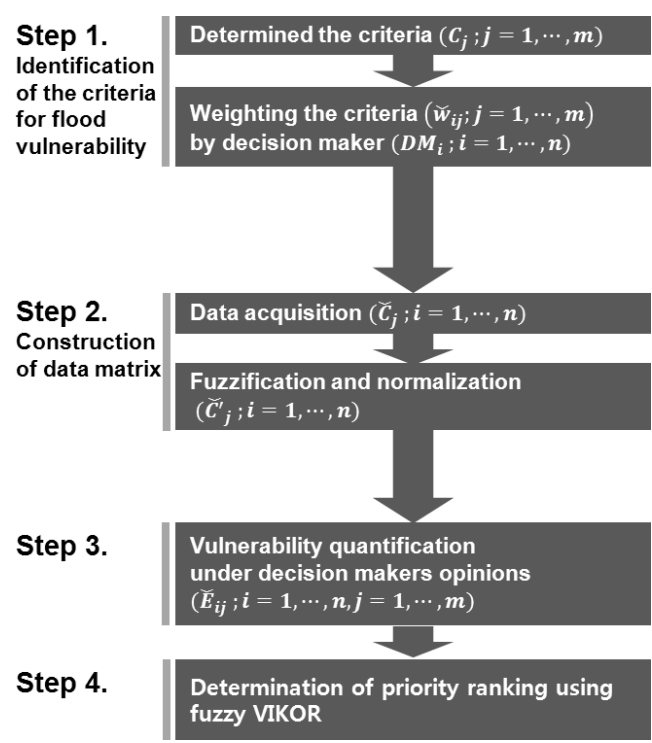

Figure 1. Study procedure for group decision making.

and flood hazards. This step was completed through a threeround individual interview.

In the second step, the data matrix was formulated using data collection and processing. The collected data from domestic public institutions served as the selected factor data. For example, population data on the number of households and asset values were obtained from the National Statistical Office (Statistics Korea: KOSTAT), and rainfall data were obtained from the Korea Meteorological Administration. The majority of the remaining data were collected from the South Korean national water information system. The probabilities of the flood levels of river sections were calculated using the frequency analysis model developed by Jun and Kim (2011). The collected data on the criteria were fuzzified and standardized. Step 3 is to quantify flood vulnerability using each weight set from each decision-making group. The sites for each participant group were prioritized individually using data collection and processing in Step 3. In step 4, the flood vulnerability priorities were derived using the VIKOR method.

\subsection{Flood vulnerability}

To identify, this study adopted the results of Lee et al. (2013) as shown in Table 1. This study had been considered critical social, economic and environmental vulnerability factors based on PSIR (pressure-state-impact-response) framework which excludes the driving force of DPSIR (driving-forcepressure-state-impact-response) framework. It is the reason why the indicators of $D$ (driving force) are widely applicable in the whole study area, and thus its value of each alternative are not distinguishable. Each attribute of PSIR is briefly described below.
Pressure leads to environmental awareness of flood risk. In turn these pressures affect the state of the environment, which refers to various flood-related circumstances and their subsequent ability to support the demands placed on. Changes in state may have an impact on human health, ecosystems, biodiversity, amenity value and financial value. The impacts may be expressed in terms of the level of harm caused by the flood. The response demonstrates the efforts by society to solve the problems identified by the assessed impacts, such as policy measures and planning actions.

Under this PSIR framework, the flood vulnerability formula can be defined as follows:

$\mathrm{FVI}=\sum \mathrm{PVul} \times w_{\mathrm{p}}+\mathrm{SVul} \times w_{\mathrm{s}}+\mathrm{IVul} \times w_{\mathrm{I}}+\mathrm{RVul} \times w_{\mathrm{R}}$,

where alignPVul, SVul, IVul and RVul are the values of pressure, state, impact and response components, respectively, which are the aggregated values of each criterion combined with the weights.

\subsection{Data fuzzification}

The real values of natural flood hazard data cannot be expressed and then obtained with ease. In addition, the technique of measurement and count have some uncertainty due to the investigation time and value averaging process. Therefore, this study applied fuzzy logic to construct data matrix for estimating flood vulnerability.

All collected data can be transformed into fuzzy membership functions using their distribution forms. Fuzzy membership functions are triangular, trapezoidal, Gaussian, generalized bell etc. From all collected data, it can be concluded that triangular type reflect well as shown in Fig. 2 and be simply calculated. Thus, all data were transformed to triangular fuzzy numbers (TFNs) in this study.

The weight sets are determined from the Delphi survey which uses fuzzy linguistic variables. Linguistic logic is useful to define the opinion of each decision maker. Table 2 presents the fuzzy linguistic variables and TFNs for the weighting. Seven linguistic variables - very low, low, medium low, medium, medium high, high, and very high were introduced.

\subsection{Fuzzy VIKOR method}

The fuzzy VIKOR method was developed by Opricovic (2011) to solve problems in an uncertain environment, in which both the criteria and weights may comprise fuzzy sets. To formally construct a fuzzy decision matrix, a typical 
Table 1. Criteria for flood vulnerability and summary of weighting.

\begin{tabular}{|c|c|c|c|c|c|c|c|c|}
\hline Category & Criteria (measure) & VL & $\mathrm{L}$ & ML & M & MH & $\mathrm{H}$ & $\mathrm{VH}$ \\
\hline Social factors & & 0 & 6 & 26 & 7 & 5 & 0 & 0 \\
\hline \multirow[t]{3}{*}{ Pressure } & & 2 & 12 & 9 & 6 & 3 & 10 & 2 \\
\hline & Population growth ratio (\%) & 0 & 6 & 13 & 3 & 19 & 3 & 0 \\
\hline & Population (number) & 2 & 4 & 0 & 9 & 15 & 7 & 7 \\
\hline \multirow[t]{4}{*}{ State } & & 0 & 12 & 11 & 10 & 9 & 2 & 0 \\
\hline & Residential and industrial area ratio (\%), & 4 & 4 & 8 & 9 & 18 & 1 & 0 \\
\hline & Population density $\left(\mathrm{man} / 1 \mathrm{~km}^{2}\right)$ & 0 & 3 & 8 & 12 & 0 & 17 & 4 \\
\hline & Number of social overhead capital, cultural properties and natural monuments (number) & 6 & 10 & 8 & 5 & 12 & 0 & 3 \\
\hline \multirow[t]{2}{*}{ Impact } & & 0 & 7 & 4 & 12 & 13 & 8 & 0 \\
\hline & Annual casualties and sufferers due to floods and disasters (number/year) & 1 & 0 & 0 & 3 & 16 & 16 & 8 \\
\hline \multirow[t]{3}{*}{ Response } & & 5 & 14 & 9 & 8 & 6 & 2 & 0 \\
\hline & Number of flood and disaster prevention institutions (number/year) & 2 & 3 & 6 & 13 & 9 & 8 & 3 \\
\hline & Number of government officials for flood and disaster mitigation (number) & 1 & 3 & 9 & 8 & 12 & 7 & 4 \\
\hline Economic factors & & 0 & 2 & 14 & 15 & 8 & 5 & 0 \\
\hline \multirow[t]{2}{*}{ Pressure } & & 8 & 10 & 10 & 6 & 8 & 2 & 0 \\
\hline & Gross regional domestic product (KRW) & 0 & 1 & 15 & 14 & 9 & 4 & 1 \\
\hline \multirow[t]{4}{*}{ State } & & 2 & 2 & 11 & 15 & 7 & 2 & 5 \\
\hline & Urban area ratio $(\%)$ & 1 & 2 & 8 & 13 & 7 & 10 & 3 \\
\hline & Self-reliance ratio of finance $(\%)$ & 0 & 8 & 12 & 10 & 11 & 1 & 2 \\
\hline & Property value(KRW) & 5 & 3 & 9 & 3 & 10 & 11 & 3 \\
\hline \multirow[t]{2}{*}{ Impact } & & 2 & 6 & 6 & 7 & 5 & 16 & 2 \\
\hline & Annual flood damage (number/year) & 1 & 0 & 0 & 0 & 5 & 24 & 14 \\
\hline \multirow[t]{2}{*}{ Response } & & 0 & 11 & 12 & 6 & 7 & 6 & 2 \\
\hline & Annual recovery and preparation costs for floods and disasters (KRW/year) & 0 & 1 & 5 & 13 & 15 & 7 & 3 \\
\hline Environmental factor & & 0 & 0 & 2 & 0 & 12 & 4 & 26 \\
\hline \multirow[t]{5}{*}{ Pressure } & & 3 & 4 & 13 & 9 & 6 & 7 & 2 \\
\hline & Increased ratio of daily maximum precipitation $(\%)$ & 0 & 7 & 13 & 1 & 9 & 11 & 3 \\
\hline & Increased ratio of $1 \mathrm{~h}$ rainfall intensity $(\%)$ & 1 & 0 & 9 & 14 & 4 & 5 & 11 \\
\hline & Increased ratio of summer rainfall $(\%)$ & 4 & 9 & 9 & 11 & 4 & 7 & 0 \\
\hline & Watershed slope (deg) & 8 & 18 & 4 & 9 & 5 & 0 & 0 \\
\hline \multirow[t]{3}{*}{ State } & & 0 & 8 & 6 & 12 & 9 & 5 & 4 \\
\hline & Peak flow of the 200 year floods & 0 & 2 & 17 & 8 & 5 & 9 & 3 \\
\hline & River stage of the 200 year floods & 0 & 6 & 12 & 6 & 7 & 7 & 6 \\
\hline \multirow[t]{3}{*}{ Impact } & & 0 & 6 & 4 & 12 & 11 & 9 & 2 \\
\hline & Annual number of floods (number/year) & 0 & 1 & 4 & 9 & 17 & 10 & 3 \\
\hline & Flood inundation area $\left(\mathrm{km}^{2}\right)$ & 1 & 0 & 0 & 13 & 11 & 12 & 7 \\
\hline \multirow[t]{3}{*}{ Response } & & 2 & 12 & 11 & 9 & 3 & 7 & 0 \\
\hline & Number of flood mitigation infrastructures (number) & 2 & 7 & 9 & 12 & 8 & 6 & 0 \\
\hline & River improvement ratio (\%) & 0 & 5 & 8 & 12 & 10 & 5 & 4 \\
\hline
\end{tabular}

fuzzy MCDM problem can be expressed in matrix form as

$$
\begin{gathered}
\widetilde{\mathbf{D}}=\begin{array}{c}
A_{1} \\
A_{2} \\
\vdots \\
A_{3}
\end{array}\left[\begin{array}{cccc}
C_{1} & C_{2} & \ldots & C_{3} \\
\tilde{x}_{11} & \tilde{x}_{12} & \ldots & \widetilde{x}_{1 n} \\
\widetilde{x}_{21} & \widetilde{x}_{22} & \ldots & \widetilde{x}_{2 n} \\
\vdots & \vdots & \vdots & \vdots \\
\tilde{x}_{m 1} & \widetilde{x}_{m 2} & \ldots & \widetilde{x}_{m n}
\end{array}\right], \\
i=1,2, \cdots, m ; j=1,2, \cdots, n . \\
\widetilde{W}=\left[\widetilde{w}_{1}, \widetilde{w}_{2}, \cdots, \widetilde{w}_{n}\right], j=1,2, \cdots, n,
\end{gathered}
$$

where $\tilde{x}_{i j}$ is the rating of alternative $A_{i}$ with respect to $C_{j}$, $\widetilde{w}_{j}$ is the importance weight of $j$ assigned by the criterion, and $\widetilde{x}_{i j}$ and $\widetilde{w}_{j}$ are linguistic variables denoted by the TFNs.

The fuzzy VIKOR method includes the following steps:
1. Determine the fuzzy best value $\left(\mathrm{FBV}, \tilde{f}_{j}^{*}\right)$ and the fuzzy worst value (FWV, $\tilde{f}_{j}^{-}$) of all criterion functions,

$$
\tilde{f}_{j}^{*}=\widetilde{x}_{i j}, j \in B ; \tilde{f}_{j}^{-}=\widetilde{x}_{i j}, j \in C .
$$

2. Compute the values $\widetilde{S}_{i}$ and $\widetilde{R}_{i}$,

$$
\begin{aligned}
& \widetilde{S}_{i}=\sum_{j=1}^{n} \widetilde{w}_{j}\left(\tilde{f}_{j}^{*}-\widetilde{x}_{i j}\right) /\left(\tilde{f}_{j}^{*}-\widetilde{f}_{j}^{-}\right), \\
& \widetilde{R}_{i}=\widetilde{w}_{j}\left(\widetilde{f}_{j}^{*}-\widetilde{x}_{i j}\right) /\left(\tilde{f}_{j}^{*}-\widetilde{f}_{j}^{-}\right),
\end{aligned}
$$

where $\widetilde{S}_{i}$ refers to the separation measure of $A_{1}$ from the best fuzzy value and $\widetilde{R}_{i}$ is the separation measure of $A_{i}$ from the worst fuzzy value. 


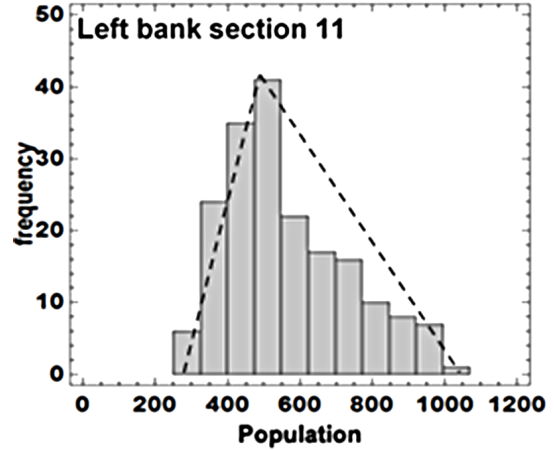

Figure 2. Fuzzy membership function with data distribution.

Table 2. Linguistic variables and fuzzy membership function for the importance of weight.

\begin{tabular}{lr}
\hline Importance & Membership function \\
\hline Very low(VL) & $(0.0,0.0,0.1)$ \\
Low(L) & $(0.0,0.1,0.3)$ \\
Medium low(ML) & $(0.1,0.3,0.5)$ \\
Medium(M) & $(0.3,0.5,0.7)$ \\
Medium high(MH) & $(0.5,0.7,0.9)$ \\
High(H) & $(0,7,0.9,1.0)$ \\
Very high(VH) & $(0.9,1.0,1.0)$ \\
\hline
\end{tabular}

3. Calculate the values $\widetilde{S}^{*}, \widetilde{S}^{-}, \widetilde{R}^{*}, \widetilde{R}^{-}$, and $\widetilde{Q}_{i}$,

$$
\begin{aligned}
& \widetilde{S}^{*}=\widetilde{S}_{i}, \widetilde{S}^{-}=\widetilde{S}_{i}, \\
& \widetilde{R}^{*}=\widetilde{R}_{i}, \widetilde{R}^{-}=\widetilde{R}_{i},
\end{aligned}
$$

where the index $\widetilde{S}_{i}$ is the maximum majority rule, the index $\widetilde{R}_{i}$ is the minimum individual regret of an opponent strategy, and $v$ is the weight of the strategy of the maximum group utility, with $v=0.5$ being a typical situation.

$$
\widetilde{Q}_{i}=\frac{v\left(\widetilde{S}_{i}-\widetilde{S}^{*}\right)}{\widetilde{S}^{-}-\widetilde{S}^{*}}-\frac{(1-v)\left(\widetilde{R}_{i}-\widetilde{R}^{*}\right)}{\widetilde{R}^{-}-\widetilde{R}^{*}}
$$

4. Defuzzify TFN $\widetilde{Q}_{i}=\left(Q_{i}^{l}, Q_{i}^{m}, Q_{i}^{u}\right)$ and rank the alternatives $(\widetilde{N})$ by the index $\mathrm{Q}_{i}$,

$\operatorname{Crisp}(\widetilde{N})=\left(Q_{i}^{l}+2 Q_{i}^{m}+Q_{i}^{u}\right) / 4$.

\section{Study area and decision-making groups}

\subsection{The southern Han River}

The southern Han River is an upper tributary of the Han River, which is located in the middle of the Korean peninsula; it includes Yeoju, which is the satellite city of Seoul.
The basin area and river length are approximately $5400 \mathrm{~km}^{2}$ and $95.4 \mathrm{~km}$, respectively; they extend from the Chungju regulating dam to the Paldang dam. The area includes six administrative districts: Gwangju, Yangpyeong, Yeoju, Icheon, Chungju, and Eumseong. Various development plans have been devised due to rapid population growth. However, the basin has experienced frequently repeated flood damages. During the last decade, the flood damage in the study area was estimated to be more than USD 150 million; the average annual flood damage in Gwangju is USD 13 million.

This study employed a reach-based areal approach to include probabilistic flood risk values. In this study, the river reach was divided into constant lengths, and its corresponding watershed was developed based on its Digital Elevation Model (DEM), as shown in Fig. 3.

\subsection{Participant decision-making groups}

An extensive range of relevant stakeholders must get involved in sustainable flood management. For example, the government has the prime responsibility of ensuring the safety and well-being of its citizens. Therefore, all relevant departments at the national, state, district, and municipal levels are closely related to flood management strategies. Scientific institutions, universities, communities, NGOs, and engineering consultants can provide useful information for compromising solutions. Each of these categories also exhibits a significant diversity of opinions (Morss et al., 2005).

In South Korea, the Ministry of Land, Infrastructure, Transport and Tourism (MLIT), which plans and manages the country's major rivers, are responsible for the flood management of large river basins. MLIT operates a dedicated flood control office for the four large basins. Although no direct management body for flood disaster management exists, the Ministry of Security and Public Administration, which is responsible for the country's total safety management, and the Ministry of Environment, which is responsible for environment management, are associated with the management of river basins and environmental pollution caused by floods. Several research institutes are also concerned with water resources, including the $\mathrm{K}$-water research institute, which is affiliated with the publicly owned water resource corporation, and government-funded research institutes, such as the Institute of Construction Technology, the National Disaster Management Institute, and the National Institute of Environmental Research. In addition, professional experts who specialize in water resource management and are affiliated with universities, engineering companies, and private research institutes, also address problems associated with floods.

Therefore, the opinions in this study were collected by conducting a questionnaire survey of people from these various institutions and organizations. Because it is difficult to obtain a consensus building for a weight set among various stakeholders and experts, this study assumed that each participator represents the group with which they are affiliated. 


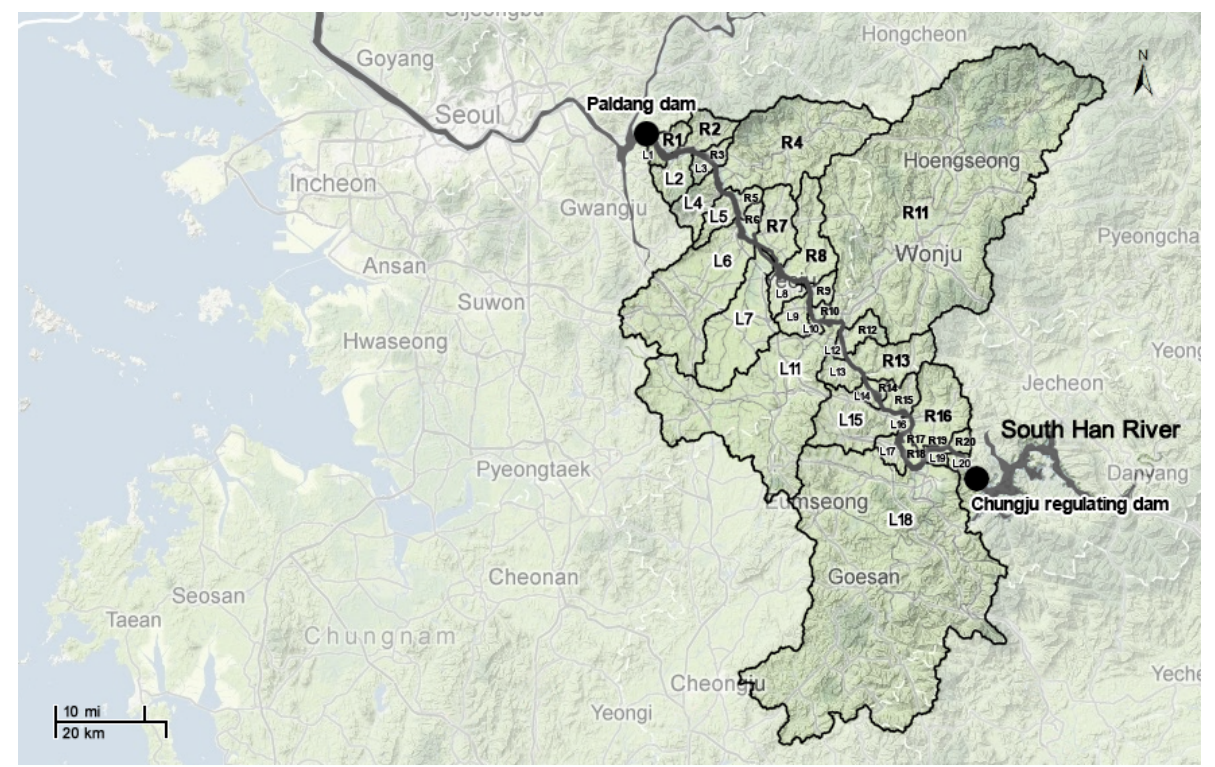

Figure 3. Study area and watershed section.

That is, each interviewee assumed to represent each individual organization associated with important flood-related business organizations was assumed to be the opinion of the entire group.

We conducted focused interviews with 44 experts who were either senior managers or experienced group facilitators. The participator had experience in flood risk management. The majority (92\%) of the participants possessed master's degree-level or doctoral-level training. They worked in the following sectors: government-funded research institutes $(34 \%)$, private engineering companies $(23 \%)$, research centers $(16 \%)$, and universities (27\%). The age distribution of the respondents ranged from 20 to 60 years, with the majority $(84 \%)$ of participants ranging between 30 and 49 years of age.

\section{Application}

\subsection{Identification of the criteria and their weights (Step 1)}

Since identifying the appropriate criteria is very crucial in vulnerability study, this study approached the systematic hierarchy structure for sustainability. The criteria were divided into three characteristic groups: social, economic, and hydrologic components. The intent was to provide supporting results for appropriate policies by forming groups of similar properties.

The research staff established a draft criteria list based on the three components and distributed it to the decisionmaking groups during the first-round survey of the Delphi procedure. For the flood vulnerability assessment, the par- ticipants completed the survey in which they expressed their opinions regarding the importance of factors. Their responses were analyzed to develop 24 criteria, as shown in Table 1. The difference of opinions among the participating decisionmaking groups was not significant, which reveals similar opinions about the criteria that need to be reflected in the calculation of flood vulnerability. Detailed opinions about the necessity of factors were collected in the second Delphi round. These opinions immediately indicated the importance of factor evaluation for assigning the weight value. Each group determined the value of the weights for the importance of the criteria using linguistic variables. The research staff subsequently analyzed and distributed the summary of the third Delphi round, and the participants reconsidered the weights. As a result, relatively compromising weights were derived. This Delphi process was described in the previous study (Lee et al., 2013).

The Delphi process is known for its ability to effectively collect the opinions of decision makers; however, decisionmaking groups have a subjective tendency to assess importance based on the analysis of the collected weights. The results of the selected weight may exhibit significant differences due to the tendencies of the decision-making groups. For example, the histogram of the weight for a particular decision-making group is shown in Fig. 4. G6 determined that the weights only use two variables, $\mathrm{MH}$ and $\mathrm{H}$ (Fig. $4 \mathrm{~b}$ ), whereas G3 (Fig. 4a) presented a diversity of opinions. In Fig. $4 \mathrm{c}$ and d, G8 used $\mathrm{M}$ for the lowest value and weighted the high level as MH, H, and VH. Conversely, G12 selected primarily low-level values as VL, L, and ML. G30 typically selected middle-level weights, whereas G38 used various weight levels. Both groups used an extensive range of 


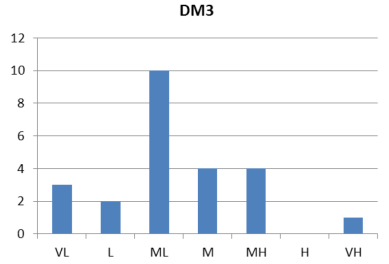

(a) Decision making group $3(\mathrm{G} 3)$

DM8

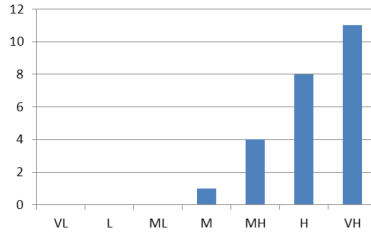

(c) Decision making group $8(\mathrm{G} 8)$

DM18

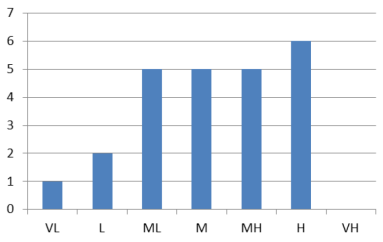

(e) Decision making group $18(\mathrm{G} 18)$
DM6

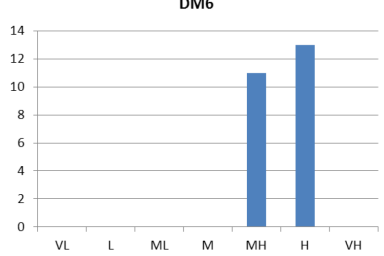

(b) Decision making group 6(G6)

DM12

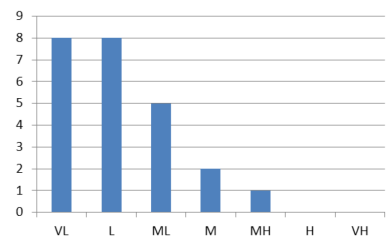

(d) Decision making group 12(G12)

DM22

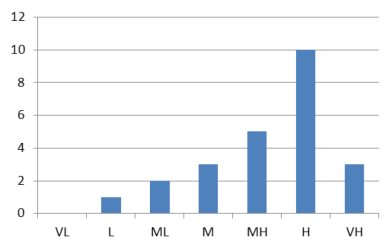

(f) Decision making group 22(G22)

Figure 4. Analysis of linguistic weight of each decision making group.

choices (Fig. 4e and f), which suggests that each group has distinct individuality.

Therefore, the collected weight sets of each decision maker were standardized as

$W_{i}^{\prime}=\frac{\left(W_{i}-W_{\min }\right)}{\left(W_{\max }-W_{\min }\right)}$,

where $W_{\max }$ and $W_{\min }$ are the maximum and minimum values of the set of weights $\left(W_{i}, i=1, \ldots, n\right)$, respectively.

For example, a comparison of the distribution between the raw and standardized data for the weights for characteristic factors is presented in Fig. 5. Regarding the social factors, ML (medium low) received the most ballots for weights, followed by M (medium) and L (low). However, the majority of ballots were altered to L (low) by standardization.

Regarding the economic factor of the weights, $\mathrm{M}$ and ML have many more ballots compared with the remaining weights. The standardizing weights of the economic factor were distributed over an extensive range between $\mathrm{L}$ and $\mathrm{H}$ (high). The most influential weight value was the diverted L through standardization. VH (very high) received the most votes for hydrologic factors, followed by $\mathrm{MH}$ (medium high). Using standardization, the most weight was predominantly distributed as ML. The standardized results of the collected weights for the criteria were mainly distributed between $\mathrm{L}$ and ML (Fig. 6).
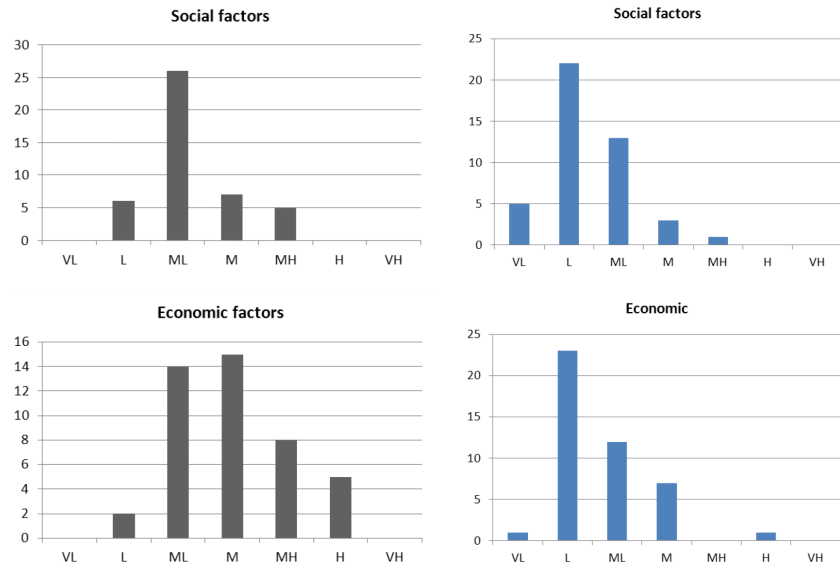

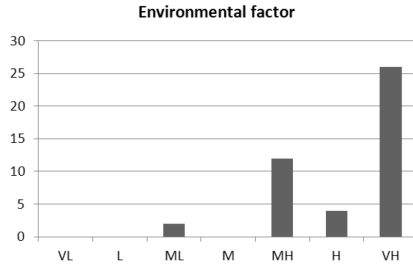

(a) Raw data

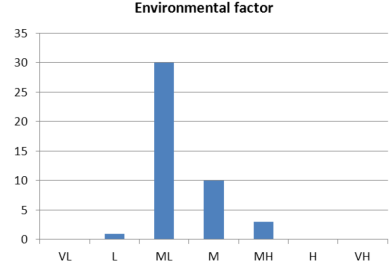

(b) Standardized data
Figure 5. Comparison of original and standardized data.
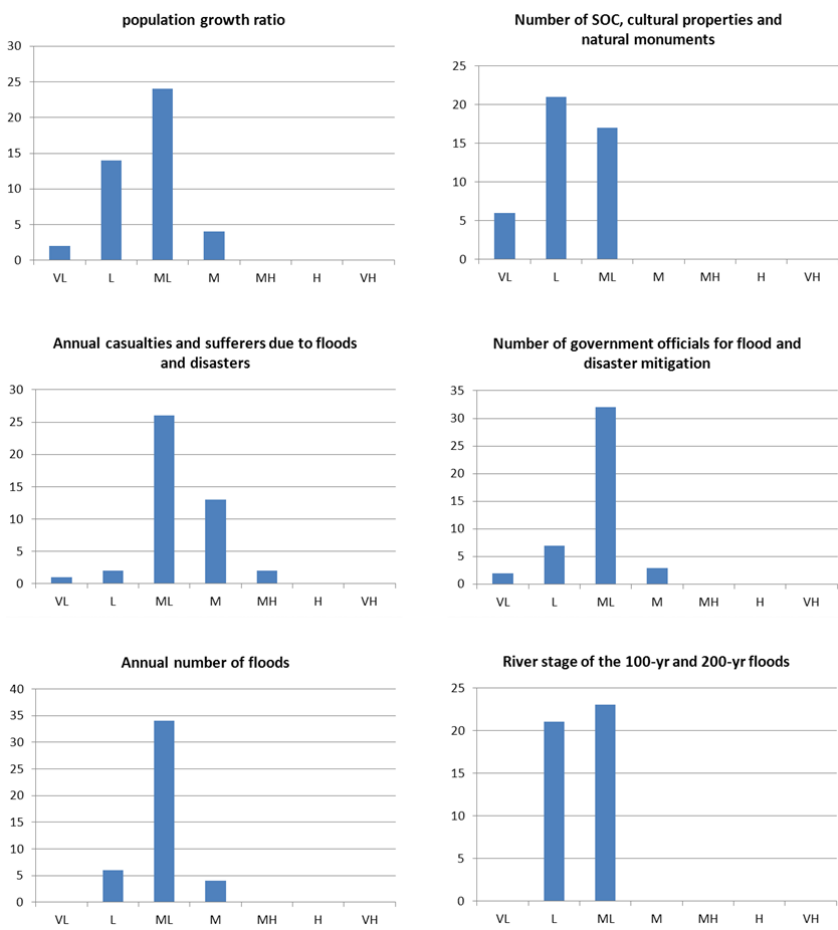

Figure 6. Standardized results of collected weights for criteria.

\subsection{Construction of the data matrix (Step 2)}

The performance value of all sections for all criteria were collected from reliable institutions, such as Statistics Ko- 

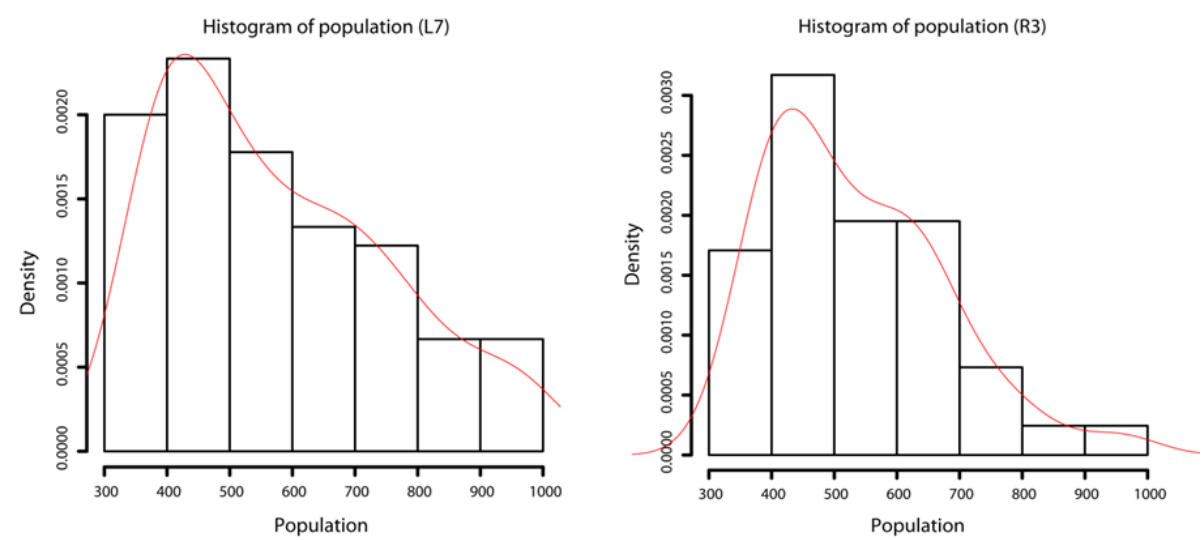

Figure 7. Examples for probability density function using R-statistics software.

Table 3. Evaluation results.

\begin{tabular}{lrrrlrrr}
\hline Alternative & $S$ & $R$ & $Q$ & Alternative & $S$ & $R$ & $Q$ \\
\hline R1 & 15 & 12 & 14 & L1 & 7 & 5 & 6 \\
R2 & 13 & 13 & 12 & L2 & 17 & 18 & 18 \\
R3 & 9 & 1 & 8 & L3 & 9 & 10 & 10 \\
R4 & 11 & 10 & 10 & L4 & 12 & 15 & 16 \\
R5 & 9 & 9 & 9 & L5 & 19 & 19 & 19 \\
R6 & 16 & 18 & 18 & L6 & 6 & 13 & 11 \\
R7 & 8 & 11 & 11 & L7 & 3 & 4 & 4 \\
R8 & 5 & 1 & 5 & L8 & 18 & 20 & 20 \\
R9 & 14 & 17 & 16 & L9 & 14 & 14 & 14 \\
R10 & 17 & 16 & 17 & L10 & 20 & 11 & 13 \\
R11 & 1 & 1 & 1 & L11 & 2 & 3 & 2 \\
R12 & 20 & 20 & 20 & L12 & 16 & 17 & 17 \\
R13 & 19 & 19 & 19 & L13 & 13 & 16 & 15 \\
R14 & 7 & 7 & 7 & L14 & 8 & 8 & 8 \\
R15 & 12 & 14 & 13 & L15 & 15 & 12 & 12 \\
R16 & 18 & 15 & 15 & L16 & 11 & 9 & 9 \\
R17 & 4 & 1 & 4 & L17 & 10 & 7 & 7 \\
R18 & 6 & 8 & 6 & L18 & 1 & 1 & 1 \\
R19 & 3 & 1 & 3 & L19 & 5 & 6 & 5 \\
R20 & 2 & 1 & 2 & L20 & 4 & 2 & 3 \\
\hline
\end{tabular}

rea, the Korea Meteorological Administration, the National Emergency Management Agency, the Water Management Information System, the local government, the National Geographic Information Institute, and the Han River flood control office. We assumed that if a province exhibits a value or extremely low variability, one value is used. The collected data were spatially analyzed, and the collected proxies were fuzzified using the TFN concept. Each TFN was statistically derived from the probability density function (PDF) using Rstatistics software, as shown in Fig. 7.

\subsection{Vulnerability identification for individual decision group (Step 3)}

In this step, the flood vulnerability for each decision group is evaluated using Eq. (9), and a performance matrix is con- structed, as shown in Eq. (10). In this assessment, we employed the same sources of criteria to identify the TFN

$$
\begin{aligned}
& \widetilde{V}=\sum_{j=1}^{m} \widetilde{w}_{j} \times \widetilde{c}_{j}, \\
& \text { Value } \\
& V_{1}\left[\begin{array}{ccc}
\widetilde{V}_{11} & \cdots & \widetilde{V}_{m 1} \\
\vdots & \ddots & \vdots \\
V_{k} & \ddots & \widetilde{V}_{m k}
\end{array}\right],
\end{aligned}
$$

$$
\begin{aligned}
& \text { Criteria value } \\
& =C_{1}\left[\begin{array}{ccc}
\tilde{c}_{11} & \cdots & \widetilde{c}_{n 1} \\
\vdots & \ddots & \vdots \\
C_{n} & \cdots & \tilde{c}_{n k}
\end{array}\right]
\end{aligned}
$$

$$
\left[\begin{array}{ccc}
G_{1} \text { Weight } \\
\widetilde{w}_{11} & \cdots & G_{m} \\
\vdots & \ddots & \widetilde{w}_{1 m} \\
\widetilde{w}_{n 1} & \cdots & \widetilde{w}_{n m}
\end{array}\right],
$$

where $\widetilde{w}$ denotes the weight sets of each group and $\widetilde{c}$ denotes the values of the criteria per unit area. They are expressed as TFNs $\left(x_{1}, x_{2}, x_{3}\right)$. The values of $\widetilde{V}$ are calculated for each group by the Weighted Sum Model (WSM). For example, the results of area R4 are shown in Fig. 8. The figure revealed a substantial difference among the opinions of each group. Some groups (G2, G10, G16, G18, G27, G36, G39, and G41) exhibited a large range of values. Conversely, the results of other groups (G4, G7, G13, G21, G22, G26, G31, G40, and G42) were confined within a narrow range. Groups G5, G12, and G19 had a high upper bound (approximately 1) compared to the other groups, whereas G2 had an uncharacteristic lower bound on 0 . 


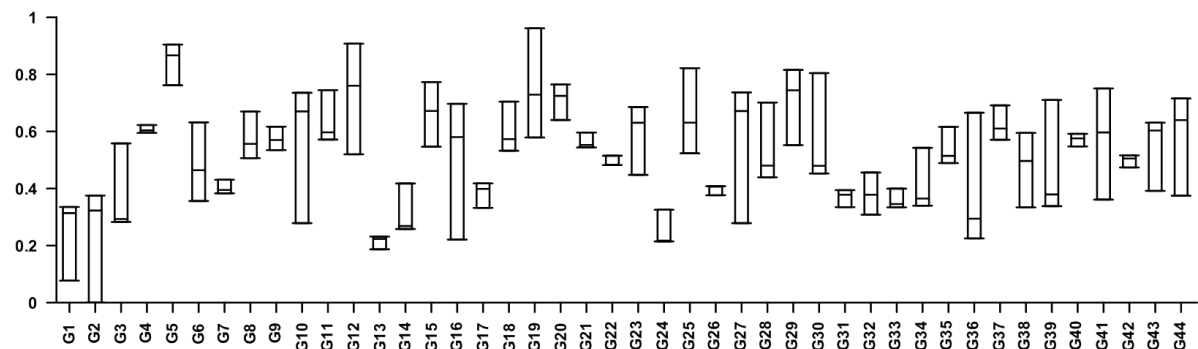

Figure 8. Determined value for R4.
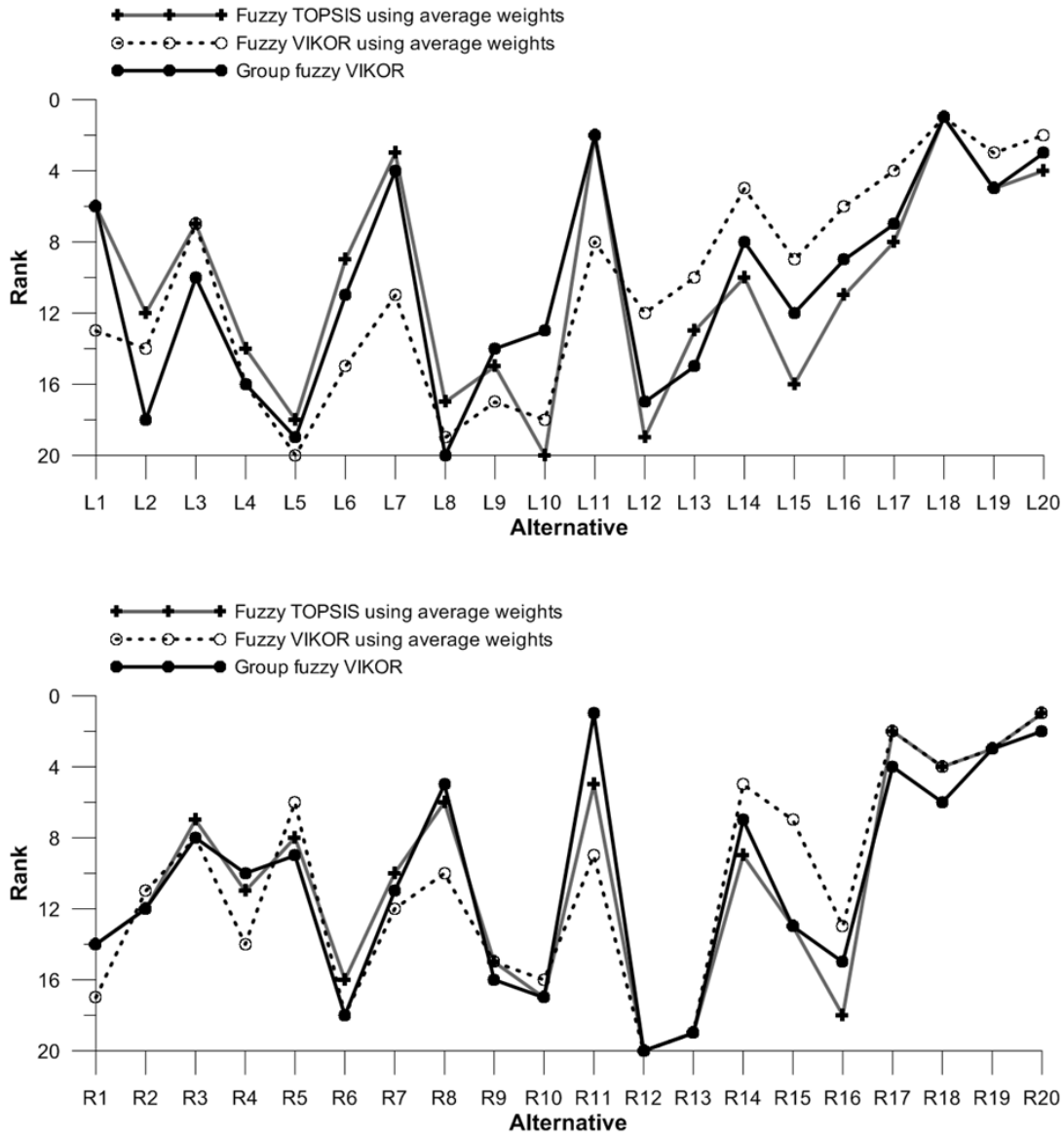

Figure 9. Comparison of assessed ranks by group fuzzy MCDM and typical MCDM approaches.

\subsection{Determination of the priority ranking using the fuzzy VIKOR method (Step 4)}

This step was intended to apply the fuzzy VIKOR GDM method to the preceding results of each group. Because the evaluated results are represented by TFNs, the priority was determined by a defuzzification value using Eq. (14). Defuzzification is another process that is used to transform a TFN into a crisp number. We used the following simple de- fuzzification method by Bojadziev and Bojadziev (1997):

$G_{\widetilde{A}}(\widetilde{x})=\frac{\mathrm{x}_{1}+2 \mathrm{x}_{2}+\mathrm{x}_{3}}{4}$.

$S, R$, and $Q$ were calculated to derive the preference ranking of the alternatives, as shown in Table 3. In this study, the priority ranks of the alternatives were determined by the $Q$ value to comprise two different types of regrets, i.e., $S$ and $R$ values. Based on the results of the fuzzy VIKOR GDM method, R11, R20, and R19 in the right bank and L18, L11, L20, and L7 in the left bank were adopted to the vulnerable area. S, R, and Q had similar ranking patterns for the 
Table 4. Comparison of evaluated ranks by GDM approaches.

\begin{tabular}{|c|c|c|c|c|c|c|c|c|c|}
\hline Alternative & Borda & Condorcet & Copeland & $\begin{array}{r}\text { Group } \\
\text { fuzzy } \\
\text { VIKOR }\end{array}$ & Alternative & Borda & Condorcet & Copeland & $\begin{array}{r}\text { Group } \\
\text { fuzzy } \\
\text { VIKOR }\end{array}$ \\
\hline R1 & 17 & 15 & 17 & 14 & L1 & 13 & 11 & 13 & 6 \\
\hline $\mathrm{R} 2$ & 14 & 13 & 14 & 12 & L2 & 18 & 16 & 18 & 18 \\
\hline R3 & 10 & 10 & 10 & 8 & L3 & 12 & 12 & 12 & 10 \\
\hline $\mathrm{R} 4$ & 13 & 14 & 13 & 10 & L4 & 11 & 13 & 11 & 16 \\
\hline $\mathrm{R} 5$ & 9 & 9 & 9 & 9 & L5 & 15 & 16 & 15 & 19 \\
\hline R6 & 15 & 17 & 15 & 18 & L6 & 6 & 6 & 6 & 11 \\
\hline R7 & 5 & 1 & 5 & 11 & L7 & 2 & 1 & 2 & 4 \\
\hline R8 & 1 & 1 & 1 & 5 & L8 & 14 & 14 & 14 & 20 \\
\hline R9 & 11 & 11 & 11 & 16 & L9 & 10 & 9 & 10 & 14 \\
\hline $\mathrm{R} 10$ & 16 & 17 & 16 & 17 & L10 & 20 & 19 & 20 & 13 \\
\hline $\mathrm{R} 11$ & 8 & 8 & 8 & 1 & L11 & 3 & 1 & 3 & 2 \\
\hline $\mathrm{R} 12$ & 20 & 19 & 20 & 20 & L12 & 17 & 16 & 17 & 17 \\
\hline $\mathrm{R} 13$ & 19 & 19 & 19 & 19 & L13 & 16 & 15 & 16 & 15 \\
\hline $\mathrm{R} 14$ & 7 & 7 & 7 & 7 & L14 & 8 & 9 & 8 & 8 \\
\hline $\mathrm{R} 15$ & 12 & 11 & 12 & 13 & L15 & 19 & 19 & 19 & 12 \\
\hline R16 & 18 & 16 & 18 & 15 & L16 & 9 & 8 & 9 & 9 \\
\hline $\mathrm{R} 17$ & 3 & 1 & 3 & 4 & L17 & 7 & 7 & 7 & 7 \\
\hline R18 & 6 & 6 & 6 & 6 & L18 & 1 & 1 & 1 & 1 \\
\hline R19 & 4 & 1 & 4 & 3 & L19 & 5 & 5 & 5 & 5 \\
\hline R20 & 2 & 1 & 2 & 2 & L20 & 4 & 1 & 4 & 3 \\
\hline
\end{tabular}

Table 5. Determined Spearman's correlation coefficients.

\begin{tabular}{|c|c|c|c|c|c|c|}
\hline \multicolumn{7}{|l|}{ Left-side bank } \\
\hline & Borda & Condorcet & Copeland & Group fuzzy VIKOR & Fuzzy TOPSIS & Fuzzy VIKOR \\
\hline Borda & - & $97.3 \%$ & $100.0 \%$ & $77.0 \%$ & $83.0 \%$ & $54.0 \%$ \\
\hline Condorcet & - & - & $97.3 \%$ & $80.1 \%$ & $85.6 \%$ & $53.9 \%$ \\
\hline Copeland & - & - & - & $77.0 \%$ & $83.0 \%$ & $54.0 \%$ \\
\hline Group fuzzy VIKOR & - & - & - & - & $87.7 \%$ & $74.8 \%$ \\
\hline Fuzzy TOPSIS & - & - & - & - & - & $67.5 \%$ \\
\hline Fuzzy VIKOR & - & - & - & - & - & - \\
\hline \multicolumn{7}{|l|}{ Right-side bank } \\
\hline & Borda & Condorcet & Copeland & Group fuzzy VIKOR & Fuzzy TOPSIS & Fuzzy VIKOR \\
\hline Borda & - & $96.1 \%$ & $100.0 \%$ & $85.5 \%$ & $90.3 \%$ & $80.0 \%$ \\
\hline Condorcet & - & - & $96.1 \%$ & $80.6 \%$ & $85.4 \%$ & $76.6 \%$ \\
\hline Copeland & - & - & - & $85.5 \%$ & $90.3 \%$ & $80.0 \%$ \\
\hline Group fuzzy VIKOR & - & - & - & - & $96.0 \%$ & $85.0 \%$ \\
\hline Fuzzy TOPSIS & - & - & - & - & - & $88.2 \%$ \\
\hline Fuzzy VIKOR & - & - & - & - & - & - \\
\hline
\end{tabular}

alternatives, whereas R8, L6, and L10 exhibited significantly different results for $S, R$, and $Q$.

For comparison, the rank of the alternatives was estimated by the fuzzy TOPSIS method and fuzzy VIKOR method using the average weight value of the participating groups. Figure 9 presents the assessing ranks. L18 of the left bank was the most vulnerable area because it yielded the highest values of the three approaches, whereas the most vulnerable area of the right bank differed among the approaches. R11, which is the most vulnerable area based on the fuzzy VIKOR GDM method, ranked 5th for the fuzzy TOPSIS method and 9th for the fuzzy VIKOR method. In the case of the test area, the results of the fuzzy TOPSIS method were analogous to our GDM approach; however, the fuzzy VIKOR method yielded different results. 
The deduced priorities shown in Table 4 facilitate the comparison of the evaluation results of the Borda, Condorcet, and Copeland methods, which are representative GDM methods that have been extensively used, with the fuzzy VIKOR GDM method proposed in this study. The evaluation rating results of the Borda, Condorcet, and Copeland methods were similar but differed significantly from the evaluation rating results of the fuzzy VIKOR GDM method.

In the case of the right bank, area R8 was ranked as the 5th most vulnerable area using the fuzzy VIKOR GDM method; however, it is ranked first for the remaining methods. R11 ranked first for the fuzzy VIKOR GDM method and 8th with the remaining methods. However, L7 and L11, which exhibited high vulnerability, ranked high for all methods, whereas L8 and L11, which exhibited low vulnerability, had significantly different rankings. Spearman's correlation coefficients were calculated to analyze the correlations among these evaluation methods, as shown in Table 5. The estimated coefficients exceeded approximately 75 percent, demonstrating that the proposed fuzzy VIKOR GDM method is generally correlated with the other MCDM methods.

\section{Conclusions}

This study used the fuzzy VIKOR GDM method to spatially quantify flood vulnerability. The motivation behind this approach is the need for a method that considers the differences of opinions among groups, which becomes evident when collecting information about the weighted value of criteria in a survey. We applied the fuzzy VIKOR method to overcome the limitations of the classical vote-based GDM methods. We compared the evaluated rankings from the fuzzy VIKOR GDM method with those from fuzzy TOPSIS and fuzzy VIKOR. The classical GDM ranks are derived by averaging all ranks from the Borda, Condorcet, and Copeland methods.

In the case of the left bank, the GDM approach with the fuzzy VIKOR method typically yielded results similar to those obtained with the existing methods for the areas ranked as highly vulnerable, whereas significantly different results were obtained for the areas that are ranked low in vulnerability. Conversely, in the case of the right bank, all evaluation methods yielded different results for the high-vulnerability areas, whereas similar results were obtained for the lowvulnerability areas. These evaluation results were obtained because the collected criteria data respond sensitively to the weighted values, as a minimal difference exists between the areas with low numbers for the left bank and a minimal difference exists between the areas with high numbers for the right bank. The evaluated priorities can change significantly depending on the applied decision-making method.

Thus, the GDM approach with the fuzzy VIKOR method can provide useful results for MCDM because it is an evaluation method that actively reflects the opinions of various groups and considers uncertainty in input data. In the next study, the focus will be put on composition of factors to assess flood vulnerability with regional capability and characteristics. Furthermore, the relationship between the flood vulnerability developed in this study and real flood damages should be investigated in the future. Then the future flood damage can be approached by flood vulnerability coupling with climate change scenarios.

Acknowledgements. This study was financially supported by the Basic Science Research Program of the National Research Foundation of Korea (NRF), which is funded by the Ministry of Education (NRF-2013R1A1A2060942).

Edited by: H. Kreibich

Reviewed by: three anonymous referees

\section{References}

Afshar, A., Mariño, M. A., Saadatpour, M., and Afsahr, A.: Fuzzy TOPSIS multi-criteria decision analysis applied to Karun reservoirs system, Water Resour. Manage., 25, 545-563, 2011.

Akter, T. and Simonovic S. P.: Aggregation of fuzzy views of a large number of stakeholders for multi-objective flood management decision-making, J. Environ. Manage., 77, 133-143, 2005.

Bojadziev, G. and Bojadziev, M.: Fuzzy logic for business, finance, and management, World Scientific Publishing Co Pte Ltd, Singapore, 69 pp., 1997.

Chang, T. H. and Wang, T. C.: Using the fuzzy multi-criteria decision making approach for measuring the possibility of successful knowledge management, Inform. Sciences, 179, 355-370, 2009.

Chen, C. T.: Extension of the TOPSIS for group decision-making under fuzzy environment, Fuzzy Set. Syst., 114, 1-9, 2000.

Chen, V. Y. C., Lien, H.-P., Liu, C.-H., Liou, J. J. H., Tzeng, G.-H., and Yang, L.-S.: Fuzzy MCDM approach for selecting the best environment-watershed plan, Appl. Soft Comput., 11, 265-275, 2011.

Chu, T. C.: Selecting plant location via a fuzzy TOPSIS approach, Int. J. Adv. Manuf. Tech., 20, 859-864, 2002.

Chu T. C. and Lin Y. C.: A fuzzy TOPSIS method for robot selection, Int. J. Adv. Manuf. Technol., 21, 284-290, 2003.

Chung, E. S. and Kim, Y.: Development of fuzzy multi-criteria approach to prioritize locations of treated wastewater use considering climate change scenarios, J. Env. Manage., 146, 505-516, 2014.

Copeland, A. H.: A reasonable social welfare function. Seminar on Applications of Mathematics to Social Sciences, University of Michigan, Ann. Arbor, November 1951, 1951.

Danube Flood Risk Project: Stakeholder oriented flood risk assessment for the Danube floodplains, Danube Flood Risk Project, available at: http://www.danube-floodrisk.eu/, last access: 2 April 2015

Fu, G.: A fuzzy optimization method for multi-criteria decision making: An application to reservoir flood control operation, Expert Syst. Appl., 34, 145-149, 2008. 
Jun, K. S. and Kim, J. S.: The Four Major Rivers Restoration Project: Impacts on river flows, KSCE J. Civil Eng., 15, 217224, 2011.

Jun, K. S., Chung, E. S., Kim, Y. G., and Kim, Y.: A fuzzy multicriteria decision approach to flood risk vulnerability in South Korea by considering climate change impacts, Expert Syst. Appl., 40, 1003-1013, 2013.

Kim, Y. and Chung, E. S.: Fuzzy VIKOR approach for assessing the vulnerability of the water supply to climate change and variability in South Korea, App. Math. Model, 37, 9419-9430, 2013a.

Kim, Y. and Chung, E. S.: Assessing climate change vulnerability with group multi-criteria decision making approaches: A case study with the water resources system in South Korea, Clim. Chan., 121, 301-315, 2013b.

Kim, Y. and Chung, E. S.: An index-based robust decision making framework for watershed management in a changing climate, Sci. Tot. Env., 473-474, 88-102, 2014.

Kim, Y. Chung, E. S., Jun, S. M., and Kim, S. U.: Prioritizing the best sites for treated wastewater use in an urban watershed using Fuzzy TOPSIS, Resour. Conserv. Recy., 73, 23-32, 2013.

Lee, G., Jun, K.-S., and Chung, E.-S.: Integrated multi-criteria flood vulnerability approach using fuzzy TOPSIS and Delphi technique, Nat. Hazards Earth Syst. Sci., 13, 1293-1312, doi:10.5194/nhess-13-1293-2013, 2013.

McLean, I.: The borda and condorcet principles: Three medieval applications, Soc. Choice and Welfare, 7, 99-108, 1990.

Morss, R. E., Wilhelmi, O. V., Dwonton, M. W., and Gruntfest, E.: Flood Risk, Uncertainty, and Scientific Information for Decision Making: Lessons from an Interdisciplinary Project, B. Am. Meteorol. Soc., 86, November 2005, 1593-1601, 2005.
Opricovic, S.: Multicriteria optimization of civil engineering systems (Visekriterijumska optimizacija sistema u gradjevinarstvu), Belgrade, Faculty of Civil Engineering, 302, 1998 (in Serbian).

Opricovic, S.: Fuzzy VIKOR with an application to water resources planning, Expert Syst. Appl., 38, 12983-12990, 2011.

Opricovic, S. and Tzeng, G. H.: Extended VIKOR method in comparison with outranking methods, Eur. J. Oper. Res., 178, 514 529, 2007.

Shih, H. S., Shyur, H. J., and Lee, E. S.: An extension of TOPSIS for group decision making, Math. Comput. Model., 45, 801-813, 2007.

Speller, G.: Improving community and citizen engagement in FRM decision making, delivery and flood response, R\&D Technical Report SC040033/SR3, product code SCHO1005BJTC-E-P, Bristol: Environment Agency, 4-6, 2005.

Torlak, G., Sevkli, M., Sanal, M., and Zaim, S.: Analyzing business competition by using fuzzy TOPSIS method: An example of Turkish domestic airline industry, Expert Syst. Appl., 38, 33963406, 2011.

Tsaur, S., Chang T., and Yen C.: The evaluation of airline service quality by fuzzy MCDM, Tourism Management, 23, 107-115, 2002.

William, G. L.: Strategic voting and the borda method, Public Choice, 33, 85-90, 1978.

Zhou, H. C., Wang, G. L., and Yang, Q.: A multi-objective fuzzy pattern recognition model for assessing groundwater vulnerability based on the DRASTIC system, Hydrolog. Sci. J., 44, 611618, 1999. 